\title{
Angular Momentum Sensitive Two-Center Interference
}

\author{
M. Ilchen*, L. Glaser, F. Scholz, P. Walter, S. Deinert, A. Rothkirch, J. Seltmann, and J. Viefhaus \\ Deutsches Elektronen-Synchrotron DESY, Notkestraße 85, 22607 Hamburg, Germany and \\ * present address: European XFEL GmbH, Albert-Einstein-Ring 19, 22761 Hamburg, Germany \\ P. Decleva \\ Dipartimento di Scienze Chimiche, Universita' di Trieste, Via L. Giorgieri 1, 34127 Trieste, Italy \\ B. Langer \\ Institut für Chemie und Biochemie, Freie Universität Berlin, Takustraße 3, 14195 Berlin, Germany
}

\author{
A. Knie and A. Ehresmann \\ Institut für Physik, Universität Kassel, Heinrich-Plett-Straße 40, 34132 Kassel, Germany
}

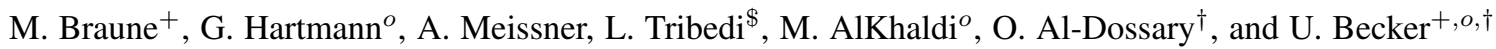 \\ Fritz-Haber-Institut der Max-Planck-Gesellschaft, Faradayweg 4-6, 14195 Berlin, Germany \\ ${ }^{\$}$ Tata Inst. of Fundamental Res., Dept. Nucl. Atom. Phys., Mumbai 400005, Maharashtra, India \\ ${ }^{\dagger}$ Department of Physics, College of Science King Saud University, Saudi Arabia \\ ${ }^{+}$present address: Deutsches Elektronen-Synchrotron DESY, Notkestraße 85, 22607 Hamburg, Germany and \\ ${ }^{\circ}$ present address: Max-Planck-Institut für Mikrostrukturphysik, Weinberg 2, 06120 Halle, Germany \\ (Dated: October 29, 2013)
}

\begin{abstract}
In quantum mechanics the Young type double-slit experiment can be performed with electrons either traveling through a double-slit or being coherently emitted from two inversion symmetric molecular sites. In the latter one the valence photoionization cross sections of homonuclear diatomic molecules were predicted to oscillate over kinetic energy almost 50 years ago. Beyond the direct proof of the oscillatory behavior of these photoionization cross sections $\sigma$, we show that the angular distribution of the emitted electrons reveals hitherto unexplored information on the relative phase shift between the corresponding partial waves through two-center interference patterns.
\end{abstract}

PACS numbers: $33.80 \mathrm{Eh}, 33.60+\mathrm{q}, 31.70-\mathrm{f}$

The Young type double-slit experiment with non-zero mass quantum objects provides a very direct view on wave-particle duality. One way to observe the fundamental quantum nature is provided by electrons being coherently emitted from two inversion symmetric molecular sites. This highly debated molecular double-slit experiment is based on the assumption that coherent electron emission from homonuclear diatomic molecules such as $\mathrm{H}_{2}, \mathrm{~N}_{2}$, and $\mathrm{O}_{2}$ leads to observable interference phenomena in the photoionization cross section of these molecules [1]. Here, the slit-distance and the wavelength of Young's classical experiment with photons correspond to the bond length and the de Broglie wavelength of the electron partial wave, respectively. It is the absence of 'whichway-information' which allows for interference. Therefore, most of the existing related studies focus on the inner shells of homonuclear diatomic molecules [2-7] to exploit the chemically localized but quantum mechanically non-localized character of the core electrons. The non-localization allows coherent electron emission from two slits which are fixed and spatially well defined. However, the fundamental formalism describing the molecular double-slit [1] was discussed for the chemically highly delocalized valence states of $\mathrm{N}_{2}$ and $\mathrm{O}_{2}$ $[1,8]$. The basic physics of that work and the numerous $\mathrm{K}$ shell studies are similar for the prediction of the oscillatory behavior of the photoionization partial cross sections $\sigma$ which is described in the following equation:

$$
\sigma_{\text {total }}=\sum_{l} \sigma_{\text {partial }}=\frac{\sigma_{\text {total }}\left(Z^{*}\right)}{(1+S)}\left[1+\frac{\sin (k R)}{k R}\right] .
$$

Here, $\sigma$ are the total and partial cross sections for which the angular momentum $l$ denotes the individual angular momentum, $Z^{*}$ is the atomic number, $k$ is the electron wave number, $R$ the molecular bond length and $S$ the overlap integral for the electrons localized at each site of the molecule. The equation implies that the molecular system absorbs a photon from both possible sites of electron excitation. Recent studies of the molecular double-slit phenomenon in the vibrational branching ratios of molecular photoionization gave first experimental evidence that the basic model of Cohen and Fano is indeed valid for the valence states [9-11]. However, the oscillation of the partial cross sections was never directly observed. In fact, the difference between almost unexplored valence and well studied K-shell double-slit experiments and the resulting impact on basic quantum physics has not been discussed so far and is subject of this work.

Challenging, regarding these oscillations, is the fact that other effects influence the cross section quite strongly and that the expected effect is small. It is interesting to mention that the experimentally observed low kinetic energy modu- 


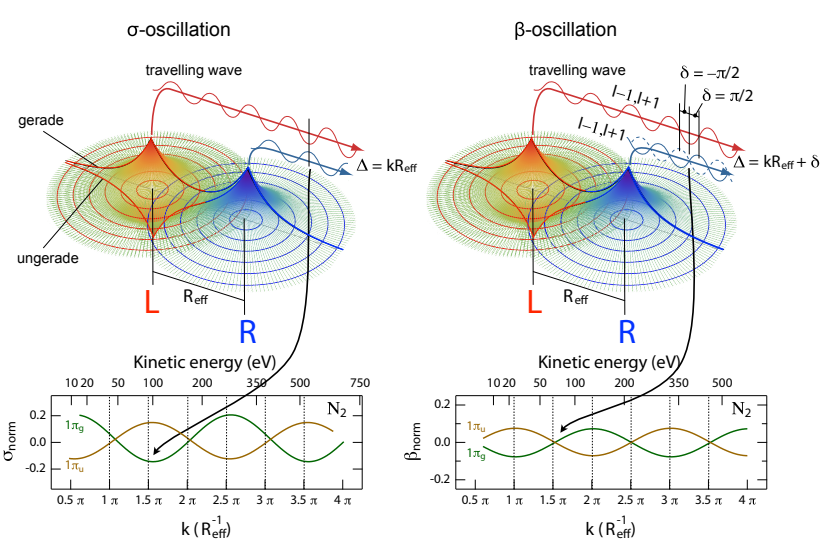

FIG. 1: Schematic representation of the coherent emission of photoelectrons from two inversion symmetric atomic sites - the molecular double-slit experiment. The left side of the figure shows the superposition of the photoelectrons from the left and the right atomic site leading to oscillations in the photoelectron partial cross sections with an oscillation frequency proportional to the inverse bond length. The right side of the figure shows the corresponding oscillation of the angular distribution anisotropy parameter $\beta$.

lation [8] does not represent the period of a real double-slit interference oscillation as originally suspected by Cohen and Fano [1]. It rather exhibits the domination of the molecular shape resonance in the cross section as our calculations and experimental data show. Meanwhile, related studies were discussing the lack of experimental data for real two-center interferences in this low photon energy range to support corresponding calculations $[12,13]$. Effects which possibly mask the pure Young type interference patterns are the scattering of the photoelectron on the neighboring atomic sites $[4,5,12]$, molecular shape resonances [14, 15], Cooper minima [16, 17], Rydberg enhanced resonances [18] and excitation of doubly excited valence-like states $[14,18]$. All of them are located in the photon energy range below $100 \mathrm{eV}$. According to our calculations and equation (1) the photoionization cross section in a much wider exciting-photon energy range, up to several hundreds of $\mathrm{eV}$, is promising to show undisturbed real molecular double-slit interference patterns. The predicted oscillation is calculated to be very shallow and the photoionization cross section rapidly decreases with increasing photon energy which underlines the experimental challenge of measuring this effect.

Following questions can be addressed by determining such $\sigma$-oscillations; besides concluding the debate of the general relevance of two-center interferences in homonuclear diatomic molecules one could use its characteristic behavior as a tool for investigating molecular properties. Most obvious is the access to the effective molecular bond length which is implicitly imprinted in the corresponding oscillation patterns [19]. A second parameter is the parity of the individual orbitals. Measuring the parity-differential cross section allows to differentiate between the 'gerade' ( $g$, bonding) and 'ungerade' ( $u$, anti-bonding) molecular orbitals which are character- istic for inversion symmetric systems. A phase difference of $\pi$ between $g$ and $u$ orbitals [1,3] results from different signs in their representation as a linear combination of one-electron atomic orbitals of the two molecular sites. Therefore, the interference oscillation phase in $\sigma$ should provide a pure measure of the molecular parity as it is qualitatively depicted by the schematic description of the coherent superposition from both sites in figure 1. More information could be carried by other photoionization parameters as i.e. the angular distribution anisotropy parameter $\beta$ (see figure 2).

The basic model for $\sigma$ is true if both partial waves, $l+1$ and $l-1$, are emitted from the two atomic sites, each from both sites (Figure 1). A completely novel perspective on twocenter interference arises if we consider the corresponding cross terms, meaning that $l+1$ is emitted from one site and $l-1$ is emitted from the other site. An additional phase shift is then introduced (right column of figure 1). This is the phase shift between the partial waves caused by the quantum defect of the preceding molecular Rydberg states. The molecular double-slit formalism and the angular distribution theory of Cooper and Zare [20] disregard this relation because they consider completely different aspects of the photoionization process. An intuitive understanding of this relationship may be obtained by first considering the origin of the photoelectron angular distribution and in a second step the implementation of the Cohen-Fano oscillations. The angular distribution is determined by the phase shift $\delta$ between the partial photoelectron waves and varies slightly with the photon energy. If the point-like emitter becomes split into two coherently emitting sites as in case of the homonuclear diatomic molecules an additional spatial phase shift affects the photoionization behavior, the so called Cohen-Fano phase shift $k R$ (see equation (1)). In first approximation, both phase shift have to be added to describe the $\beta$-oscillation being proportional to $\cos (\delta+k R)$; this is what we observe.

This interpretation is supported by studies of the $K$-shell photoionization of $N_{2}$ [3, 4, 7]. Figure 3c shows the angular distribution pattern for $N_{2} 1 s$ ionization. These data and the expected $\beta_{a b s}=2$ of the $K$-shell at higher photon energies highlight that $\beta$-oscillations appear in the near threshold regime but do not persist over larger photon energy ranges because intramolecular scattering giving rise to higher partial waves dies out and hence the corresponding phase dependence. This is different for the case of the valence orbitals of $\mathrm{N}_{2}$ and $\mathrm{O}_{2}$. They are mainly composed of their respective atomic $p$-states. In consequence, the emitted electrons after photoionization $(l \pm 1)$ are predominantly $s(l-1)$ and $d(l+1)$ partial waves. These two partial waves quantum mechanically allow the simultaneous emission of an $s$-wave on one side of the molecule and a $d$-wave emission on the other side and vice versa (figure 1). Such measurements consequently provide information on the relative phase shift between the outgoing partial waves of the emitted photoelectron. In noncoherent experiments without two-center interference, its determination requires at least three independent measurements because the minimal number of photoionization parameters is 


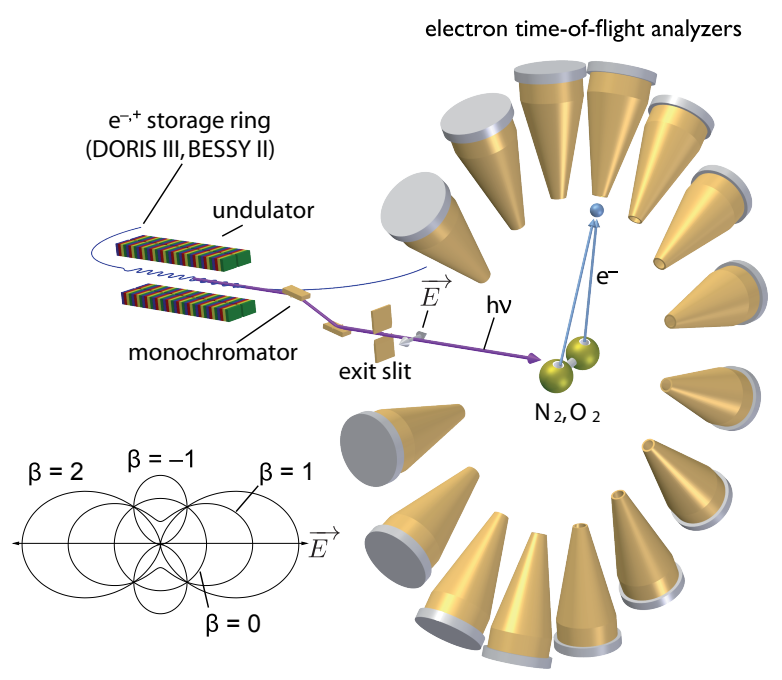

FIG. 2: Schematic description of the experimental setup and the angular distribution anisotropy parameter $\beta$. The apparatus consists of 16 independently operating time-of-flight electron spectrometers placed on a circle. The gas inlet introduces the molecules into the interaction volume of the incident photon beam and the molecular target being in the center of that circle. The photons are generated in an undulator structure of $e^{+}$and $e^{-}$storage rings, in the case of the present experiments DORIS III at DESY in Hamburg and BESSY II in Berlin, respectively. The photons are monochromatized by plane grating monochromators. The angular distribution anisotropy parameter $\beta$ is visualized at bottom left.

three, assuming LS-coupling; two partial waves and one phase shift between them (three-parameter model) [21]. In a molecular double-slit experiment, however, this phase shift becomes directly apparent by only two measurements.

A very weak pattern of maximum amplitude of $\sim 0.05 \beta$ units is expected by theory [22]. Such challenging demands of the experimental investigations necessitated the development of a new highly efficient angle resolving photoelectron spectrometer setup capable of resolving the parity states in the whole discussed photon energy range (see figure 2 ). In order to have access to the relevant wide photon energy range with a sufficiently high photon flux we applied our new spectrometer setup to the synchrotron radiation facility DORIS III at DESY in Hamburg. A similar experiment was carried out at BESSY II at the Helmholtz-Zentrum Berlin, both in Germany. The measurements at DESY were carried out at beamline BW3 $[23,24]$ which provides monochromatized synchrotron radiation in the energy range from 18 to $1500 \mathrm{eV}$ with an energy resolution of $\frac{E}{\Delta E}>1000$ over the whole presented energy range with a photon flux $>10^{12} \frac{h \nu}{\mathrm{s}}$. All measurements of our gas phase experiment were primarily performed with the new angle resolving photoelectron spectrometer setup which employed 16 independently working electron time-of-flight spectrometers aligned in the plane of the linear photon beam polarization (see figure 2). This spectrometer setup collects $\sim 4 \%$ of the full solid angle in total. Each time of flight spectrom- eter is capable of decelerating the incoming electrons using 4 independent static electric potentials within the flight tube and therefore sustaining the energy resolution sufficiently to resolve all valence states of interest even up to $600 \mathrm{eV}$. The experiment at BESSY II employed beamline UE 56/2 PGM1 which provides a similar performance as the BW3 III beamline in the energy range from 60 to $1300 \mathrm{eV}$. The spectrometer setup used at BESSY II was very comparable to the one used at DORIS III but consisting of 22 time-of-flight spectrometers. These spectrometers were providing an even better energy resolution but compromising the detection efficiency.

Enabled by the novel apparatus, we were able to prove the oscillatory structure of the $\mathrm{N}_{2}$ and $\mathrm{O}_{2}$ valence photoionization cross sections from randomly oriented molecules (figure 3 ) as they were predicted almost 50 years ago [1]. This oscillation is indeed bond length dependent. One full period of $\Delta k=2 \pi$ is spread over many hundreds of $\mathrm{eV}$ as expected from equation (1). The original description by Cohen and Fano [1] as well as our new calculations are in good agreement with the present experimental results for the showcase of nitrogen. For $\sigma$ the observed oscillation displays a phase shift of $\pi$ between the 'gerade' and 'ungerade' states of both homonuclear diatomic molecules, as expected. The proof of the $\sigma$-oscillations for four individual cases in $\mathrm{N}_{2}$ and $\mathrm{O}_{2}$ directly validates the original Cohen and Fano model of the molecular double-slit experiment. A detailed understanding of the analogous cross section oscillations in the $K$-shell and the valence shell photoionization is necessary to prove the essential difference between the two cases regarding the interference observed in the angular distribution. As discussed before, no such dependence over larger energy ranges can be expected in the $K$-shell photoionization but for the $\mathrm{N}_{2}$ and $\mathrm{O}_{2}$ valence shells (figures $3 \mathrm{~b}$ and 3 e respectively) we indeed observe an oscillatory behavior of the electron angular distribution anisotropy parameter $\beta$ over several hundred $\mathrm{eV}$. In figure 3 the values of the normalized cross sections are derived by dividing the obtained individual intensities of each parity by the sum of both contributions. Therefore, we are able to extract the oscillatory behavior of the gerade and ungerade states against each other without measuring the absolute cross sections. The obtained absolute values for the angular distribution anisotropy parameter $\beta$ [25] were first normalized by subtracting the general, non-oscillatory trend of the curves. Consistently, the oscillatory behavior of the 'gerade' and 'ungerade' states was then derived by the same procedure as for the relative cross sections. The results are consistent with the general Cohen Fano model. This model is extended by the unprecedented twocenter interference oscillation in the angular distribution of the coherently emitted electrons from two spatially separated atomic sites. These oscillations directly reflect the $l$-phase difference of the individual orbitals. For $N_{2}$ the phase shift between the $\sigma$ and the $\beta$-oscillation is $0.6 \pi$ whereas for $O_{2}$ this phase shift is close to $\pi$ matching the literature values for the $l$-phase differences of atomic nitrogen and oxygen [2631]. The orbital-averaged effective bond lengths for the valences are $0.094 \mathrm{~nm}$ and $0.084 \mathrm{~nm}$ for $N_{2}$ and $O_{2}$ respectively 

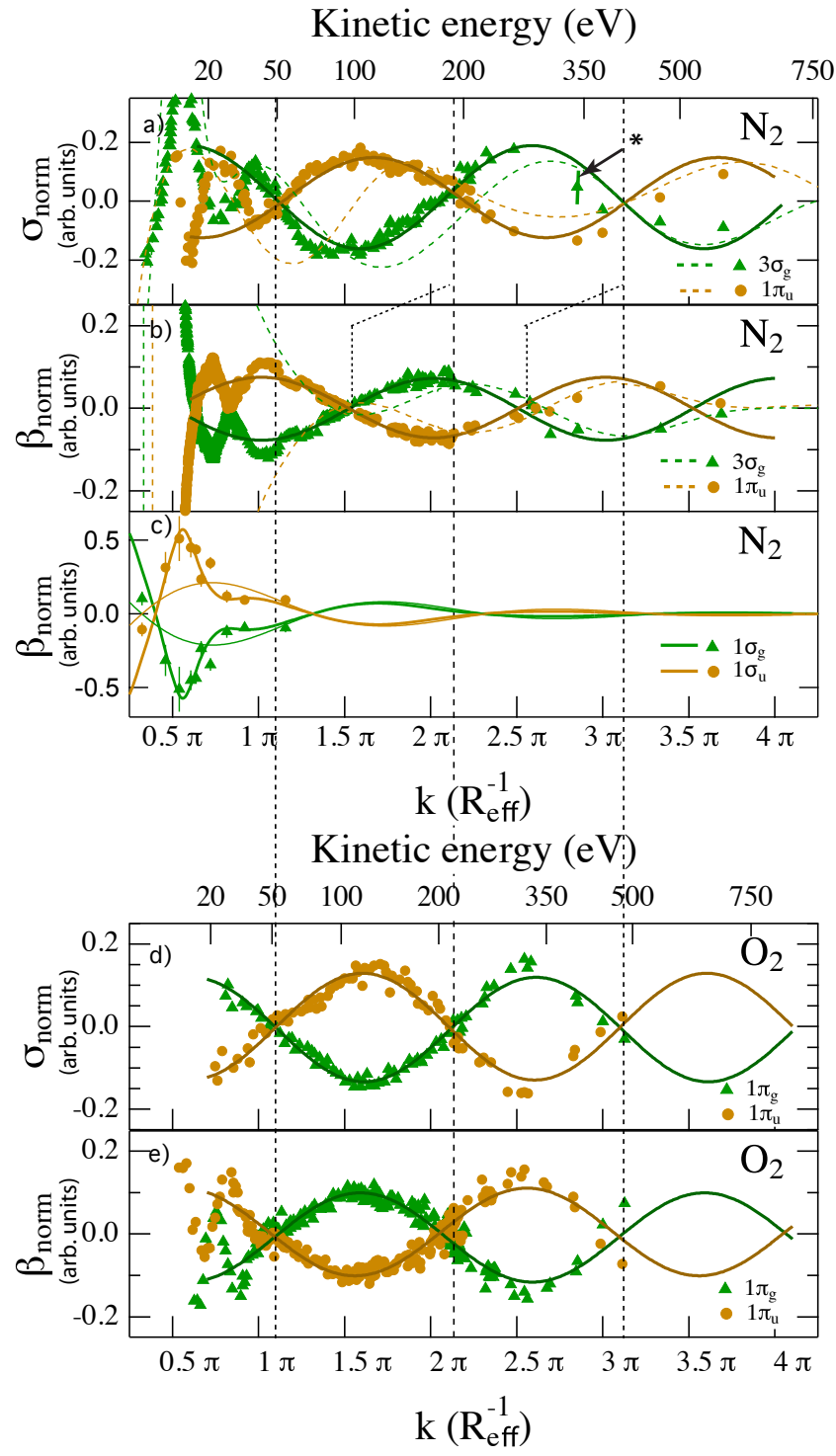

FIG. 3: Normalized experimental (symbols) and theoretical (dashed lines) partial cross sections $\sigma$ and angular distribution anisotropy parameters $\beta$ for the valence photoionization of $N_{2} 3 \sigma_{g}^{-1}, N_{2} 1 \pi_{u}^{-1}$, $\mathrm{O}_{2} 1 \pi_{g}^{-1}$ and $\mathrm{O}_{2} 1 \pi_{u}^{-1}$ displayed over the kinetic energy in $\mathrm{eV}$ and $\mathrm{k}$ $\left(R_{e f f}^{-1}\right)$. For the $O_{2} 1 \pi_{u}^{-1}$ only the $a^{4} \Pi_{u}$ state is included. These are the outer valences for both molecules with different parity. Panels (a) and (d) show the $\pi$-shifted $\sigma$-behavior for the two-center interference oscillations for the $g$ and $u$ orbitals of $N_{2}$ and $\mathrm{O}_{2}$ respectively. Panels (b) and (e) show the corresponding $\beta$-oscillations for $N_{2}$ and $\mathrm{O}_{2}$ where the respective 'gerade' and 'ungerade' states are consistently shifted by $\pi$. This phase shift is imprinted due to the inversion symmetry of the molecules and the corresponding sign change in the creation of 'ungerade' orbitals. The $K$-shell $\beta$-data of [3] is depicted in panel (c). The asterisk in panel (a) marks a typical error bar for the high kinetic energy region of $\mathrm{N}_{2}$ and $\mathrm{O}_{2}$.

which suggests that the highly delocalized valence state has a smaller effective bond length compared to the equilibrium bond lengths.

In conclusion, we have shown that the theoretical model of the molecular double-slit experiment is directly valid for the originally discussed cases of partial photoionization cross sections of molecular valence states. On this basis we were able to investigate a hitherto unobserved angular dependence of matter-wave interferences. They display the relative phase shift between the outgoing partial waves in a visually very direct way due to the coherently emitted photoelectrons from two spatially separated inversion symmetric centers. This phenomenon represents the first case which shows that Young's double-slit experiment with non-zero mass particles is essentially different for valence and core electron ionization of inversion symmetric systems.

We acknowledge the discussions with D. Rolles (DESY, Germany), M. Meyer (European XFEL, Germany) and K. Ueda (Tohoku Univ., Japan). Furthermore, we thank the DESY and HZB staff for providing smooth machine conditions and sedulous support during the beamtimes. Part of the authors are indebted to the BMBF for financial support within the FSP 301 'FLASH: Materie im Licht ultrakurzer und extrem intensiver Röntgenpulse' and the MPG-ASG at CFEL in Hamburg. UB is furthermore grateful to the 'Distinguished Scientist Fellowship Program' of the King Saud University, Riyadh (grant number 10-MAT1217-02).

[1] H. D. Cohen and U. Fano, Phys. Rev. 150, 30 (1966).

[2] D. Akoury et al., Science 318, 949 (2007).

[3] D. Rolles et al., Nature 437, 711 (2005).

[4] B. Zimmermann et al., Nature Physics 4, 649 (2008).

[5] O. A. Fojón, J. Fernández, A. Palacios, R. D. Rivarola, and F. Martín, J. Phys. B 37, 3035 (2004).

[6] E. Shigemasa, J. Adachi, M. Oura, and A. Yagishita, Phys. Rev. Letters 74, 359 (1995).

[7] X. J. Liu et al., J. Phys. B 39, 4801 (2006).

[8] J. A. R. Samson and R. B. Cairns, J. Opt. Soc. Am. 55, 1035 (1965).

[9] S. E. Canton et al., PNAS 108, 7302 (2011).

[10] E. Plésiat, P. Decleva, and F. Martín, Phys. Chem. Chem. Phys 14, 10853 (2012).

[11] U. Becker, Nature 474, 586 (2011).

[12] M. Okunishi et al., Phys. Rev. Lett. 103, 043001 (2009).

[13] A. S. Baltenkov, U. Becker, S. T. Manson, and A. Z. Msezane, J.Phys. B 45, 035202 (2012).

[14] S. H. Southworth, A. C. Parr, J. E. Hardis, and J. L. Dehmer, Phys. Rev. A 33, 1020 (1986).

[15] M. N. Piancastelli, J. Electr. Spectr. Relat. Phenom. 100, 167 (1999).

[16] J. B. Bertrand, H. J. Wörner, P. Hockett, D. M. Villeneuve, and P. B. Corkum, Phys. Rev. Letters 109, 143001 (2012).

[17] R. Della Picca, P. D. Fainstein, and A. Dubois Phys. Rev. A 84, 033405 (2011).

[18] M. Ilchen et al., J. Phys. B 45, 225102 (2012).

[19] M. Schoeffler et al., Phys. Rev. A 78, 013414 (2008).

[20] J. Cooper and R. N. Zare, J. Chem. Phys. 48, 942 (1968).

[21] U. Becker, J. Elec. Spectr. Relat. Phenom. 96, 105-115 (1998).

[22] D. Toffoli and P. Decleva, J. Phys. B 39, 2681 (2006).

[23] T. Möller, Synch. Rad. News 6, 16 (1993).

[24] C. U. S. Larsson, Nucl. Instr. and Meth. A 437, 603 (1994). 
[25] M. Ilchen, PhD Thesis TU Berlin http://opus4.kobv.de/opus4tuberlin/frontdoor/index/index/docId/3381 (2012).

[26] W. M. Kosman and S. Wallace, J. Chem. Phys. 82, 1385 (1985).

[27] S. T. Pratt, J. L. Dehmer, and P. M. Dehmer, Phys. Rev. A 36, 1702 (1987)

[28] S. T. Manson, J. Electr. Spectr. Relat. Phenom. 1, 413 (1972).
[29] C. E. Theodosiou, M. Inokuti, and S. T. Manson, Atom Data Nucl. Data 35, 473 (1986).

[30] A. Gerwer, C. Asaro, B. V. McKoy, and P. W. Langhoff, $J$. Chem. Phys. 72, 713 (1980).

[31] U. Becker and B. Langer, Phys. Scripta T78, 13 (1998). 